\title{
Arginase inhibition attenuates arteriogenesis and interferes with M2 macrophage accumulation
}

\author{
Manuel Lasch ${ }^{1,4}$, Amelia Caballero-Martinez ${ }^{1,4}$, Kerstin Troidl ${ }^{2,3}$, Irmengard Schloegl ${ }^{1}$, Thomas Lautz ${ }^{1}$ and \\ Elisabeth Deindl ${ }^{1}$
}

L-Arginine is the common substrate for nitric oxide synthases (NOS) and arginase. Whereas the contribution of NOS to collateral artery growth (arteriogenesis) has been demonstrated, the functional role of arginase remains to be elucidated and was topic of the present study. Arteriogenesis was induced in mice by ligation of the femoral artery. Laser Doppler perfusion measurements demonstrated a significant reduction in arteriogenesis in mice treated with the arginase inhibitor nor-NOHA ( $\mathrm{N}^{\omega}$-hydroxy-nor-arginine). Accompanying in vitro results on murine primary arterial endothelial cells and smooth muscle cells revealed that nor-NOHA treatment interfered with cell proliferation and resulted in increased nitrate/ nitrite levels, indicative for increased NO production. Immuno-histological analyses on tissue samples demonstrated that nor-NOHA administration caused a significant reduction in M2 macrophage accumulation around growing collateral arteries. Gene expression studies on isolated growing collaterals evidenced that nor-NOHA treatment abolished the differential expression of Icam 1 (intercellular adhesion molecule 1). From our data we conclude that arginase activity is essential for arteriogenesis by promoting perivascular M2 macrophage accumulation as well as arterial cell proliferation. Laboratory Investigation (2016) 96, 830-838; doi:10.1038/labinvest.2016.62; published online 30 May 2016

Cardiovascular diseases such as myocardial infarction or stroke are still the major cause of morbidity and death worldwide. Current options to treat affected patients are percutaneous transluminal angioplasty, percutaneous transluminal coronary angioplasty or bypass surgery. ${ }^{1}$ Aiming to develop non-invasive therapeutic options to treat such patients much effort is made to understand the molecular mechanisms of arteriogenesis. Arteriogenesis is the growth of a natural bypass and occurs as adaptive response of the body to vascular occlusion. ${ }^{1}$ It is well established that the trigger for arteriogenesis is increased laminar shear stress. ${ }^{2}$ This increased mechanical load is exerted on ECs (endothelial cells) of pre-existing arteriolar connections by the redirected blood flow caused by occlusion of a conductance artery. ${ }^{3}$ Animal models, mainly for peripheral arteriogenesis, evidenced that increased shear stress results in activation of the arteriolar endothelium with subsequent upregulation of monocyte chemoattractant protein-1 (MCP-1) as well as ICAM1 presenting the prerequisite for monocyte recruitment, adhesion, and extravasation. ${ }^{4-6}$ After extravasation, monocytes mature to macrophages, whereby M1 as well as M2 macrophages have been described to contribute to vascular remodeling. ${ }^{7}$

Interestingly, it has been shown that exogenously administrated nitric oxide $(\mathrm{NO})$ donors promote arteriogenesis. ${ }^{8,9}$ This is somehow amazing as it is well known that NO counteracts leukocyte adhesion as well as smooth muscle cell proliferation (for an overview see ${ }^{10}$ ), two essential processes in arteriogenesis. Indeed, the mechanistic function of NO for collateral artery growth still remains elusive.

In the body, $\mathrm{NO}$ can be produced by three different isoforms of the NOS enzyme: NOS1, NOS2, and NOS3 (neuronal, inducible, and endothelial NO synthase). These isoforms display differences in tissue distribution, intracellular location, molecular regulation, and enzymatic kinetics. ${ }^{11}$ All three isoforms have been shown to be involved in arteriogenesis. ${ }^{9,12-14}$ NOS1 is mainly localized in smooth muscle cells (SMCs) of collateral arteries and is upregulated during arteriogenesis. ${ }^{12}$ NOS3 presents the major form of NOS in ECs, and has a role in maintaining native collateral density. ${ }^{14}$ NOS3 enzymatic activity is critical for NO-mediated vasodilation in collateral vessels, however, is not essential for effective collateral artery growth as evidenced

\footnotetext{
Walter-Brendel-Centre of Experimental Medicine, LMU Munich, Munich, Germany; ${ }^{2}$ Max-Planck-Institute for Heart and Lung Research, Bad Nauheim, Germany and ${ }^{3}$ Department of Vascular and Endovascular Surgery, University Hospital Frankfurt, Frankfurt, Germany

Correspondence: Dr E Deindl, PhD, Walter-Brendel-Centre of Experimental Medicine, LMU Munich, Marchioninistr. 15, 81377 Munich, Germany.

E-mail: elisabeth.deindl@med.uni-muenchen.de

${ }^{4}$ These authors contributed equally to this work.
}

Received 9 November 2015; revised 5 April 2016; accepted 21 April 2016 
by histological analyses. ${ }^{9} 13$ NOS2 deficiency in contrast strongly interferes with collateral artery growth. ${ }^{9}$ As NOS2 is the major form of NOS expressed in leukocytes it is likely that leukocyte derived NO is relevant for effective arteriogenesis.

L-Arginine is the substrate for NOS, however, also presents the substrate for arginase. ${ }^{11}$ It has previously been demonstrated that arteriogenesis is reduced in animals treated with L-NAME, which was attributed to disrupted NO production. ${ }^{15}$ However, L-NAME does not only inhibit NOS but also arginase. ${ }^{16}$ There are two different isoforms of arginase, arginase 1 and arginase 2, showing different subcellular locations. Whereas arginase 1 is located in the cytosol, arginase 2 is a mitochondrial enzyme. ${ }^{11}$ The function of arginase 2 is less well characterized, however, arginase 1 has been shown to be essential for vascular cell proliferation, and to be a feature of M2 macrophages. ${ }^{11}$ We therefore asked whether arginase is essential for the process of arteriogenesis.

For our study, we employed a murine hindlimb model in which femoral artery ligation results in collateral artery growth. ${ }^{5}$ We evaluated the efficiency of the process of arteriogenesis by measuring hindlimb perfusion noninvasively by means of laser Doppler imaging (LDI) and quantified macrophage infiltration as well as Icam 1 expression in mice treated with the arginase inhibitor nor-NOHA compared with saline-treated control mice. Furthermore, we performed accessory in vitro proliferation assays on primary murine arterial ECs and SMCs and investigated NO production by means of nitrate/nitrite measurements.

\section{MATERIALS AND METHODS Reagents}

L-Lys (L-lysine monohydrochloride) and L-Arg (L-arginine) were purchased from Sigma-Aldrich (St. Louis, MO, USA), nor-NOHA from Bachem (Bubendorf, Switzerland). For proliferation assays, L-Arg-, L-Lys- phenol-free DMEM (Dulbecco's Modified Eagle's Medium; Sigma-Aldrich) was supplemented with $272 \mu \mathrm{M}$ L-Lys.

\section{Animals and Treatments}

The investigation was approved and controlled by the local ethics committee and carried out according to the guidelines of the German law for protection of animal life. Mice were kept under a $12 \mathrm{~h}$ day and night cycle with chow and water provided ad libitum. Eight to 10 weeks old C57/BL6 male mice (Charles River, Sulzfeld, Germany) were used for in vivo experiments. To inhibit arginase in vivo, mice were treated with nor-NOHA $50 \mathrm{mg} / \mathrm{kg}$ i.p. (intraperitoneal) per day dissolved in $0.9 \% \mathrm{NaCl}$ starting one day before the surgical procedure until the end of the experiment. The control group received saline only. L-Arginine was dissolved in drinking water $(5 \%, \mathrm{w} / \mathrm{v}),{ }^{17-19}$ corresponding to a dose of about $330 \mathrm{mg}$ per day per animal, and $\mathrm{pH}$ was adjusted to 7 . L-Arginine supplementation was started 3 days before the surgical procedure and administered until the end of the experiment.

\section{Femoral Artery Ligation, Laser Doppler Perfusion and Blood Pressure Measurements}

To induce peripheral arteriogenesis, C57/BL6 mice underwent unilateral femoral artery ligation (occ) of the right leg whereas the left leg was sham operated as previously described. ${ }^{5,20}$ During surgery none of the mice died and no necrosis or infection of the wound was observed during the observation period.

Blood flow measurements were conducted using the LDI technique (Moor LDI 5061 and Moor Software Version 3.01, from Moor Instruments, Remagen, Germany) as previously described. ${ }^{5,20}$ Mice were anesthetized and kept at $37^{\circ} \mathrm{C}$ body temperature in a heating chamber for $10 \mathrm{~min}$. The measurements were performed before surgery, directly after surgery, and on day 3 and day 7 after surgery. The right-to-left ratio was calculated for each mouse and background tissue values were subtracted to eliminate tissue bias.

Blood pressure was measured non-invasively by determining the tail blood pressure using a two-module system consisting of an occlusion tail cuff and a volume pressure recording sensor from CODA System (Kent Scientific, Torrington, CT, USA). The measurements were done as previously described. ${ }^{21}$ The blood pressure was measured one day after the surgical procedure and at day 7 .

\section{Tissue Sampling for Immuno-Histology and RNA Expression Analyses}

For RNA tissue sampling, the abdominal aorta was cannulated, and both hindlimbs were perfused with latex flexible compound (Chicago Latex, Chicago, IL, USA) to visualize superficial collateral arteries. Twelve hours after surgery, two superficial collateral arteries per animal per side were isolated. All samples were snap frozen in dry ice and stored at $-80^{\circ} \mathrm{C}$.

For histology tissue sampling on day 7 after the surgical procedure, mice were perfused as described above with $0.1 \%$ $(\mathrm{w} / \mathrm{v})$ adenosine (Sigma-Aldrich) and $0.5 \%(\mathrm{w} / \mathrm{v})$ bovine serum albumin (Sigma-Aldrich) dissolved in phosphatebuffered saline (PBS; PAN Biotech, Aidenbach, Germany), $\mathrm{pH} 7.4$, followed by 3\% (for cryopreservation) or $4 \%$ (for paraffin embedding) paraformaldehyde in $\mathrm{PBS}, \mathrm{pH} 7.4$, for $20 \mathrm{~min}$. For cryoconservation adductor muscles were cut and placed in $15 \%(\mathrm{w} / \mathrm{v})$ sucrose in PBS for $4 \mathrm{~h}$ and in $30 \%$ sucrose $(\mathrm{w} / \mathrm{v})$ overnight at $4{ }^{\circ} \mathrm{C}$. Muscle samples were then embedded in tissue tek (Sakura, Alphen an den Rijn, The Netherlands) and cut in $6 \mu \mathrm{m}$ sections. For paraffin embedding adductor muscles were placed in $4 \%$ paraformaldehyde for $24 \mathrm{~h}$, and after paraffin embedding cut in $5 \mu \mathrm{m}$ sections.

\section{Immuno-Histochemical Staining}

Mice received BrdU (bromodeoxyuridine, $1.25 \mathrm{mg}$ per day, i.p.; Sigma-Aldrich) dissolved in PBS for 6 consecutive days starting at the day of the surgical procedure. Sections of 
paraffin-embedded tissue were immuno-stained with an antiBrdU antibody (BD Biosciences, San Jose, CA, USA) using the BrdU In-Situ Detection Kit (BD Biosciences) according to manufacturer's protocol. Staining were analyzed with Axioscop 40 (Carl Zeiss AG, Feldbach, Switzerland). Data are shown as percent of $\mathrm{BrdU}^{+}$cells of total cells.

Cryofixed tissue sections were immuno-stained for MRC1 (Abcam, Cambridge, UK), CD68-Alexa647 (AbDSerotec, Puchheim, Germany), $\alpha$ SMA-Cy3 ( $\alpha$ smooth muscle actin; Sigma-Aldrich), or ICAM1 (Abcam), respectively, as previously described. ${ }^{7}$ Perivascular macrophages of the two superficial arteries showing the largest luminal caliber of at least three cross-sections per animal were counted by a blinded observer using a confocal microscope (Leica SP5) and image analysis was carried out with ImageJ Software (National Institutes of Health, Maryland, USA).

\section{RNA Isolation, CDNA Synthesis and qRT-PCR}

RNA was isolated from collateral arteries with Trizol (Life Technologies, Carlsbad, CA, USA). Residual genomic DNA was removed by RQ1 RNase-Free DNase (Promega, Madison, WI, USA) digestion, and RNA was purified with RNeasy MinElute columns (Qiagen, Hilden, Germany). RNA (250 ng) was reverse transcribed into cDNA using the QuantiTect Rev. Transcription Kit (Qiagen). qRT-PCR (quantitative reverse transcriptase PCR) was performed with $1 \mu \mathrm{l}$ of the 1:5 diluted cDNA per reaction and $0.5 \mu \mathrm{M}$ of each primer using Power SYBR Green Kit (Life Technologies) according to the manufacturer's protocol and run in a StepOnePlus cycler (Life Technologies). Primer sequences were as follow: $18 \mathrm{~S}$ rRNA forward 5'-GGACAGGATTGACAGATTGATAG-3', $18 \mathrm{~S}$ rRNA reverse $5^{\prime}$-CTCGTTCGTTATCGGAATTAAC-3', Icam 1 forward $5^{\prime}$-GGAGGTGGCGGGAAAGTTC- $3^{\prime}$ and Icam 1 reverse $5^{\prime}$-CTGCTGAGACCCCTCTTGC-3'. Three independent qRT-PCR reactions were performed on each template. The protocol was as follows: an initial denaturation step at $95^{\circ} \mathrm{C}$ for $10 \mathrm{~min}$ was followed by 40 cycles of denaturation $\left(95^{\circ} \mathrm{C}, 15 \mathrm{~s}\right)$, annealing and extension $\left(58^{\circ} \mathrm{C}\right.$ for Icam $1,64^{\circ} \mathrm{C}$ for $18 \mathrm{~S}$ rRNA, $1 \mathrm{~min}$ ). Melt curve analyses and agarose gels were performed to control specific amplification. Analyses were performed with the $\Delta \Delta \mathrm{Ct}$ method. Results were normalized to the expression levels of the 18S rRNA.

\section{Cell Culture}

Mouse primary coronary artery endothelial cells and mouse primary artery smooth muscle cells were purchased from Cellbiologics (Chicago, IL, USA). Cells were cultured in complete mouse endothelial cell or complete mouse smooth muscle cell medium, respectively, supplemented with growth factors supplied by Cellbiologics. Cells were grown onto $1 \%$ gelatine (PAN Biotech; SMCs) or collagen A (Biochrom AG, Berlin, Germany; ECs) coated flasks and medium was changed every two days. Passages 1 to 3 were used for experiments.

\section{BrdU Proliferation Assay}

Cell proliferation was analyzed with the BrdU proliferation kit from Roche (Basel, Switzerland). Briefly, primary arterial ECs and SMCs were seeded in a 96-well plate a $10^{4}$ cells per well for $24 \mathrm{~h}$. For starvation, medium was replaced by L-Arg-free DMEM with $1 \%$ fetal calf serum (FCS; PAN Biotech) for $24 \mathrm{~h}$. Thereafter, cells were stimulated with $800 \mu \mathrm{M}$ L-Arg with or without $800 \mu \mathrm{M}$ nor-NOHA in 2\% FCS L-Arg-free DMEM supplemented with BrdU for two days. Cells serving as controls were cultured in 2\% FCS L-Arg-free DMEM supplemented with BrdU for the same time period. Thereafter, cells were fixed and proliferation was assessed by colorimetry following manufacturer's instructions.

\section{Determination of Nitrate and Nitrite Concentrations}

Nitrate and nitrite concentrations in cell supernatants were assessed by the Nitrate/Nitrite Fluorometric Assay Kit (Cayman Chemical, An Arbor, MI, USA) according to the manufacturer's protocol. In brief, nitrate was reduced to nitrite with the nitrate reductase and nitrite concentration was determined by fluorescence with an extinction wavelength of $365 \mathrm{~nm}$ and an emission wavelength of $430 \mathrm{~nm}$ in a Tecan fluorometer (Männedorf, Switzerland).

\section{White Blood Cell Counts}

Blood samples were gained by cardiac puncture and collected in EDTA-tubes (SARSTEDT, Nümbrecht, Germany) to avoid coagulation. White blood cell counts were investigated using the Hematology analyzer Indexx ProCyte Dx ${ }^{\mathrm{TM}}$ (Indexx Laboratories, Westbrook, ME, USA).

\section{Statistical Analysis}

Statistical analyses were performed with GraphPad software PRISM6 (GraphPad Software, La Jolla, CA, USA). Data are presented as means \pm s.e.m. Results were tested for normality and statistical analyses were performed as indicated in the figure legends. Results were considered to be statistically significant at $P \leq 0.05$.

\section{RESULTS \\ Arginase Activity is Essential for Arterial Cell Proliferation and Impacts NO Production}

To determine whether arginase activity is essential for arteriolar cell proliferation, we performed proliferation assays on primary arterial ECs and SMCs. Our results evidenced that L-arginine supplementation significantly promoted proliferation of ECs as well as SMCs, which was blocked when cells were treated with the arginase inhibitor nor-NOHA concomitantly (Figures 1a and b). In the same experimental setting we investigated the level of nitrate/nitrite concentration in cell supernatant as marker for NO production. In EC cultures L-arginine treatment increased nitrate/nitrite levels, which were further elevated when nor-NOHA was present. In SMC cultures, in contrast, L-arginine treatment reduced nitrate/nitrite levels, whereas treatment of cells with 
a
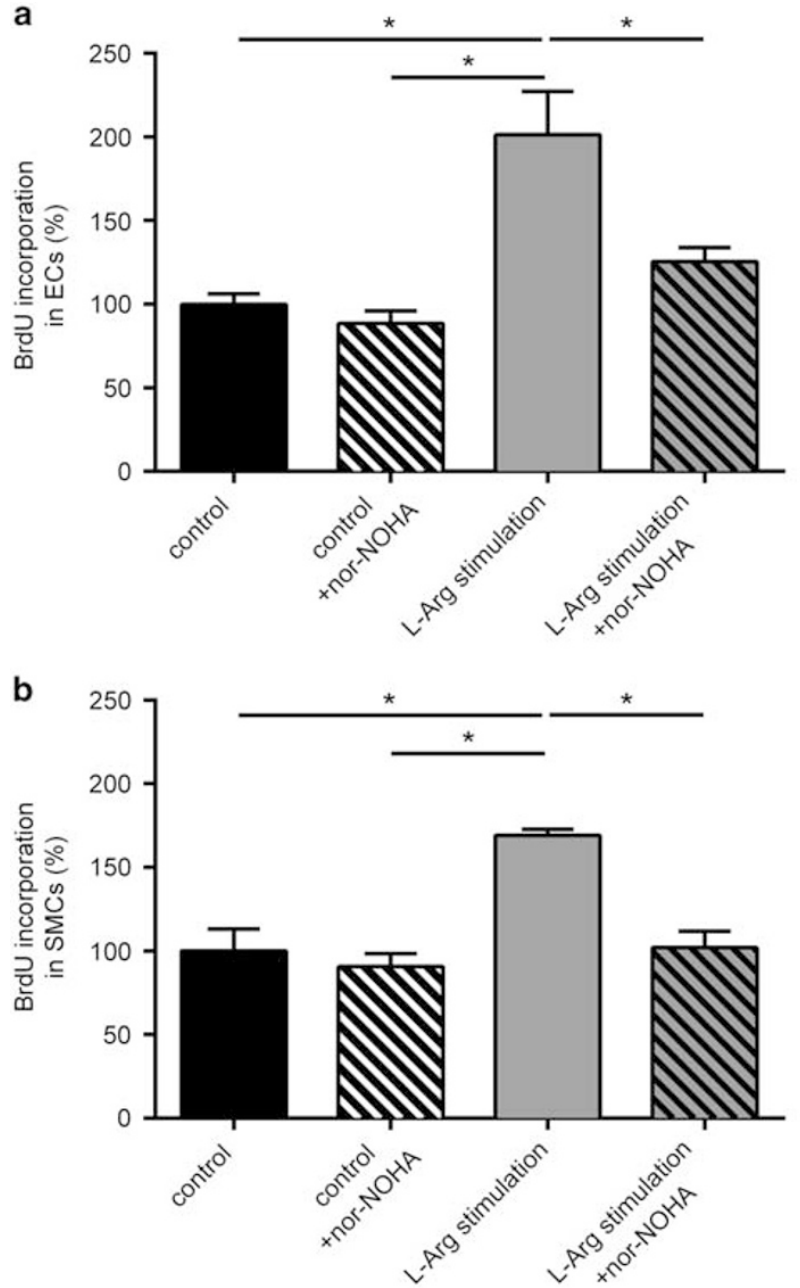

c

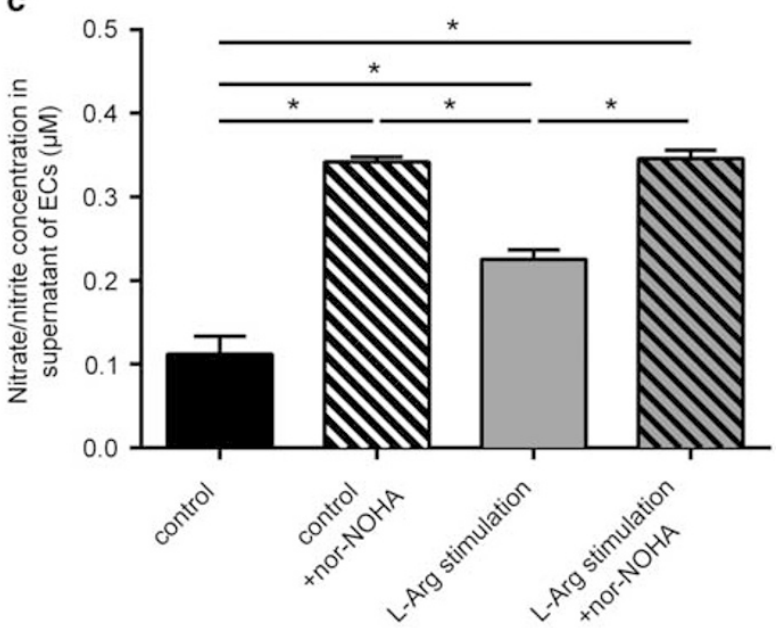

d

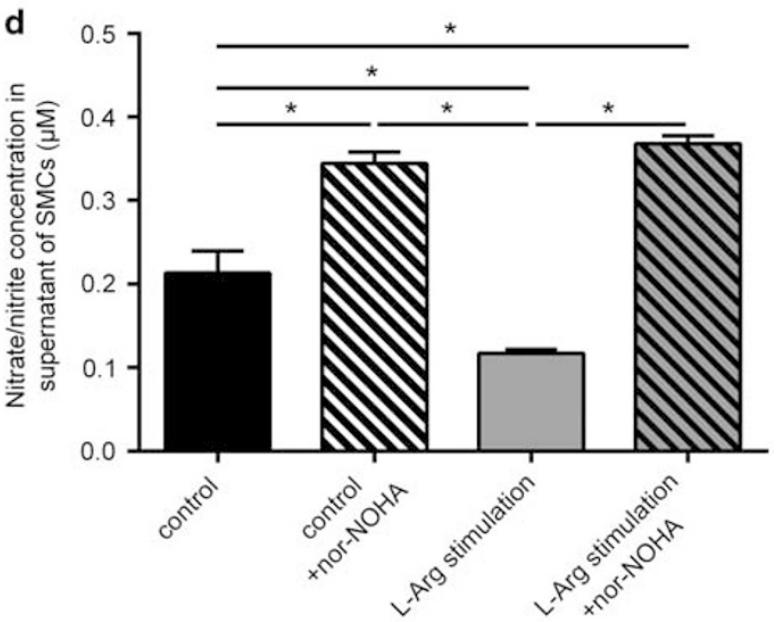

Figure 1 Proliferation assay on primary arterial ECS and SMCs, and nitrate/nitrite concentration in cell supernatants. Cells were either treated with Larginine (L-Arg) alone or concomitant or alone with nor-NOHA. (a) Bar graphs representing the percentage of BrdU incorporation in ECs and (b) SMCs. Values were normalized to control cells which were grown without L-arginine supplementation. (c) Bar graph representing the concentration of nitrate and nitrite in supernatant of ECs and (d) SMCs. Nitrate/nitrite concentration of the DMEM medium was subtracted from the total concentration. Data are means \pm s.e.m. $n=3$ per group, experiments were repeated three times. ${ }^{*} P \leq 0.05$ one-way ANOVA with Bonferroni's multiple comparison test.

L-arginine together with nor-NOHA significantly increased nitrate/nitrite levels. In control ECs and SMCs, which were grown without L-arginine supplementation, nor-NOHA treatment resulted in increased nitrate/nitrite levels as well (Figures $1 \mathrm{c}$ and $\mathrm{d}$ ).

\section{Arginase Inhibition Attenuates Arteriogenesis}

To investigate the relevance of arginase for arteriogenesis in vivo, we employed a murine hindlimb model. In this model, femoral artery ligation rapidly results in the growth of collateral arteries showing typical cork screw formation (Figure 2, left panel), whereas on the sham operated side only faint preexisting collaterals are visible (Figure 2, right panel).

To address the biological significance of arginase in arteriogenesis, we treated mice with the arginase inhibitor nor-NOHA and measured hindlimb perfusion in vivo by means of LDI. Compared with saline-treated controls, norNOHA-treated mice showed a significant reduction in perfusion recovery one week after induction of arteriogenesis by femoral artery ligation (Figures $3 \mathrm{a}$ and $\mathrm{b}$ ). To investigate whether the observed reduction in perfusion recovery was due to a reduced proliferation of vascular cells, BrdU staining was performed (Figure 3c). Our results evidenced a significant reduction in the number of BrdU-positive ECs in nor-NOHA vs saline-treated mice, whereas the number of BrdU-positive SMCs was reduced only to a minor and not significant degree (Figure 3d). To test whether L-arginine, which is the substrate for NOS as well as for arginase, might be a rate limiting substrate in arteriogenesis, another group of mice were treated with this amino acid. However, our results evidenced no significant improvement in perfusion recovery (Figures 3a and b). L-Arginine treatment did not significantly influence 

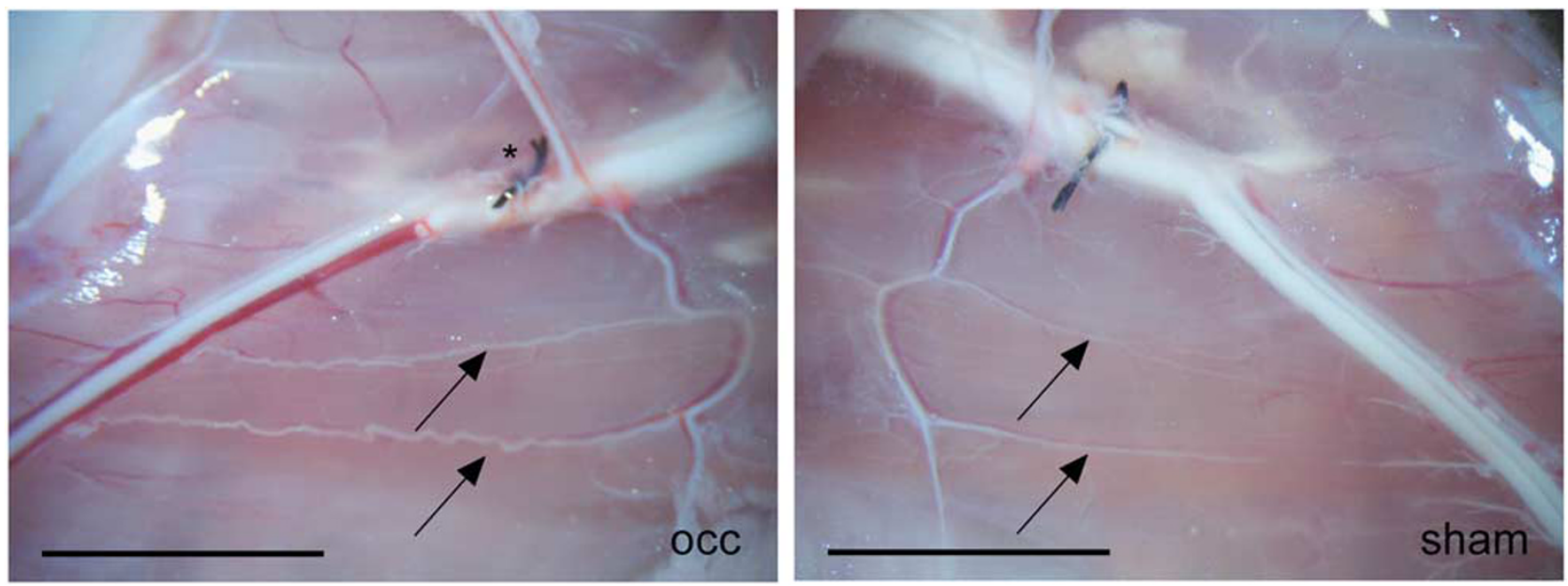

Figure 2 Characteristics of collateral vessel morphology. Photographs of superficial collateral arteries (arrows) in the adductor muscle 7 days after the surgical procedure. On the left, the femoral artery was occluded downstream of the epigastrica artery $\left(^{*}\right)$ leading to arteriogenesis. The vessels present themselves in the typical cork screw formation of growing collaterals. On the right, the femoral artery was sham operated at the same position of the femoral artery. The pre-existing collateral arteries in their resting state appear very straight and show a tiny caliber. Scale bars, $5 \mathrm{~mm}$.

the systolic, diastolic, or mean arterial blood pressure of mice (data not shown).

\section{Arginase Inhibition Interferes with Perivascular Accumulation of M2 Macrophages}

To investigate whether arginase is essential for recruitment and perivascular accumulation of leukocytes we performed gene expression studies and investigated the expression level of ICAM1, which we found to be expressed on ECs of collateral arteries $24 \mathrm{~h}$, and on ECs and SMCs at day 7 after induction of arteriogenesis. Our qRT-PCR results showed that the differential expression of Icam 1 seen $12 \mathrm{~h}$ after femoral artery ligation in saline-treated mice was abolished when mice were treated with nor-NOHA (Figures $4 \mathrm{a}$ and $\mathrm{b}$ ).

To analyze in more detail the influence of arginase inhibition on perivascular accumulation of leukocytes and in particular on macrophage subpopulations, we performed quadruple-labeled confocal microscopy on tissue samples isolated on day 7 after the surgical procedure. We therefore employed an $\alpha$ smooth muscle actin ( $\alpha \mathrm{SMA})$ marker and the macrophage activation-type specific markers CD68 and MRC1. Our immuno-histochemical analyses evidenced a significant reduction in the number of perivascular $\mathrm{CD} 68^{+}$ $/ \mathrm{MRC1}^{+} \mathrm{M} 2$-polarized macrophages in the occluded leg of nor-NOHA-treated mice (Figure 5), whereas the count of blood monocytes as well as other leukocyte subpopulations was not significantly different between nor-NOHA- and saline-treated mice (Table 1).

\section{DISCUSSION}

In our study, we analyzed whether arginases have an essential role in arteriogenesis, the growth of natural bypasses. This question is of major interest, as arginases compete with NOS for the substrate L-arginine, and $\mathrm{NO}$ synthesis has previously been shown to be relevant for effective arteriogenesis. ${ }^{9}$ To investigate the contribution of arginase in collateral artery growth, we used a murine hindlimb model for arteriogenesis. ${ }^{5,20}$

Arginase 1 and 2 have been detected in both, ECs and SMCs, however, the abundance of each isoform is different between animal species, vascular bed size, and function of blood vessel. Moreover, both isoforms of arginase have been described to be induced by various stimuli and to contribute to vessel wall remodeling. ${ }^{11}$

As the vessel wall remodeling during arteriogenesis involves the proliferation of arteriolar ECs and SMCs, we were interested whether L-arginine supplementation might foster vascular cell proliferation. We therefore cultured primary murine arterial ECs and SMCs without, and in the presence of L-arginine. Our results showed that L-arginine supplementation supported both, EC as well as SMC proliferation. Furthermore, in ECs L-arginine supplementation resulted in increased nitrate/nitrite levels, indicative for increased NO production. Indeed it has been previously been described that exogenously added L-arginine can be channeled to $\mathrm{NO}$ production by endothelial NOS3 in vitro. ${ }^{22}$ Interestingly, L-arginine supplementation resulted in decreased nitrate/ nitrite levels in supernatants of SMCs. Recent studies have shown that the neuronal isoform of NOS, NOS1, is almost not present in arteriolar ECs, but is strongly expressed in collateral arteriolar SMCs. ${ }^{12,23}$ Moreover, it has been demonstrated that arginase 1 shows the same cellular distribution as NOS1. ${ }^{24}$ Together these data strongly suggest that arginase 1 and NOS1 compete for L-arginine in arteriolar SMCs, however, that L-arginine is mainly metabolized by arginase 1 in proliferating SMCs.

To investigate whether L-arginine supplementation might foster the process of arteriogenesis, mice received L-arginine supplementation in drinking water starting three days before 
a
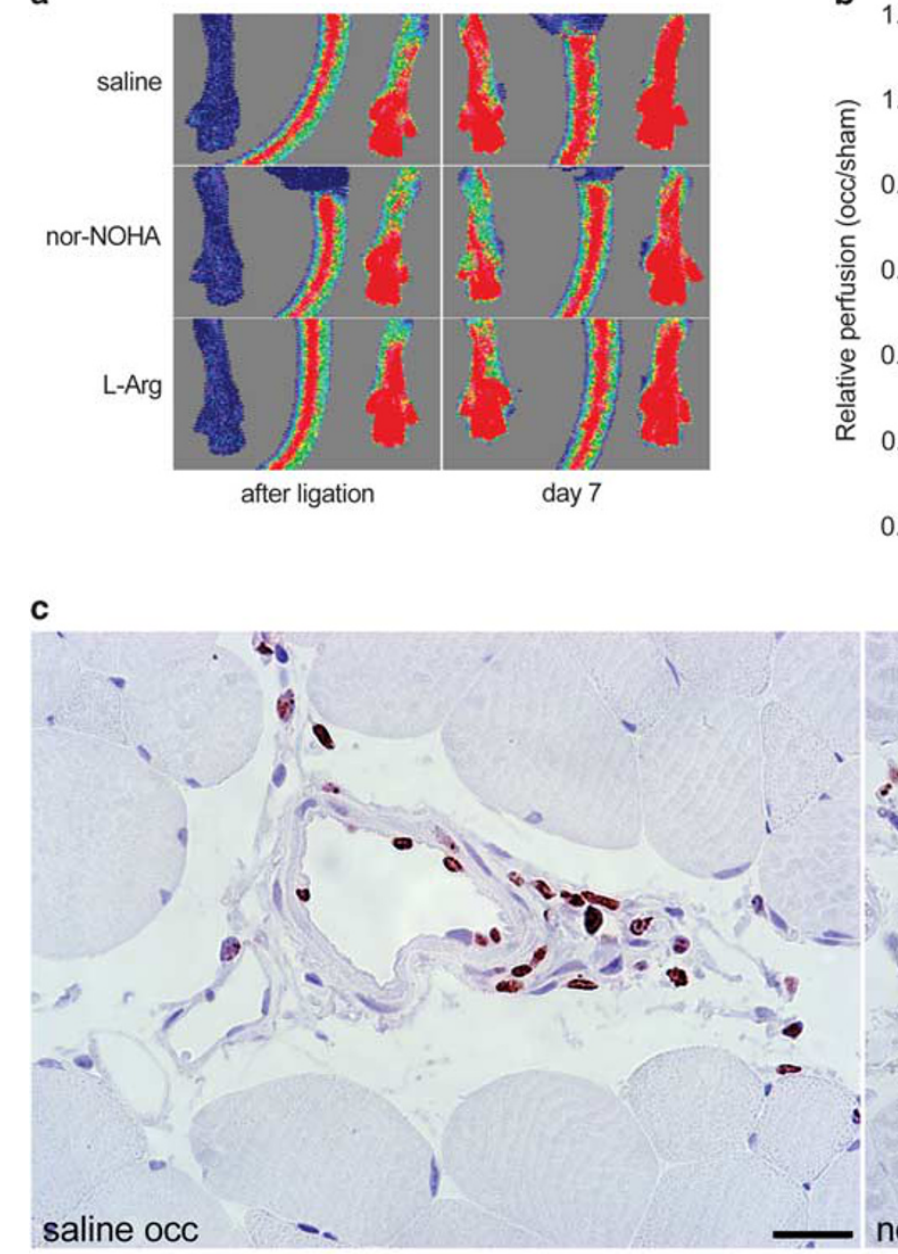

c

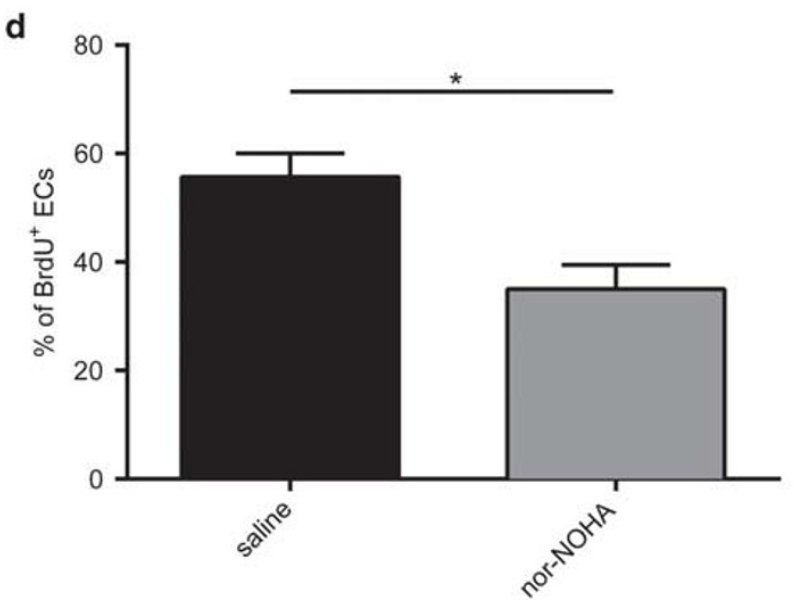

b

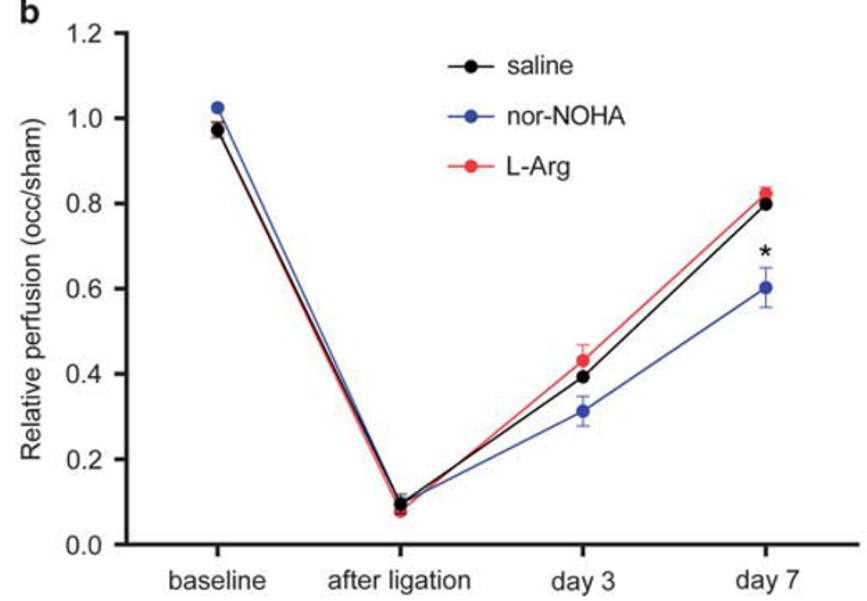

\section{d}

Figure 3 Laser Doppler analyses of mice after femoral artery ligation. (a) Representative laser Doppler flux images of mice treated with saline, L-arginine (L-Arg), or nor-NOHA, immediately after femoral artery ligation and on day 7. The images show the paws of the hindlimbs as well as the tail of mice. (b) The right-to-left ratio of blood flow in paws was calculated for each mouse of the cited groups in a time period of 7 days. Data are means \pm s.e.m., $n=6$ per group. ${ }^{*} \leq 0.05$ (vs saline-treated group) two-way ANOVA with Bonferroni's multiple comparison test. (c) Representative immuno-histochemical staining of collaterals at day 7 after induction of arteriogenesis in mice treated with saline or nor-NOHA. BrdU-positive cells are stained in brown. Scale bars, $20 \mu \mathrm{m}$. (d) Quantification of BrdU-positive ECs (left panel) and SMCs (right panel). Data are means \pm s.e.m., $n \geq 10$ per group. ${ }^{*} P \leq 0.05$ (vs salinetreated group) unpaired Student's $t$-test.

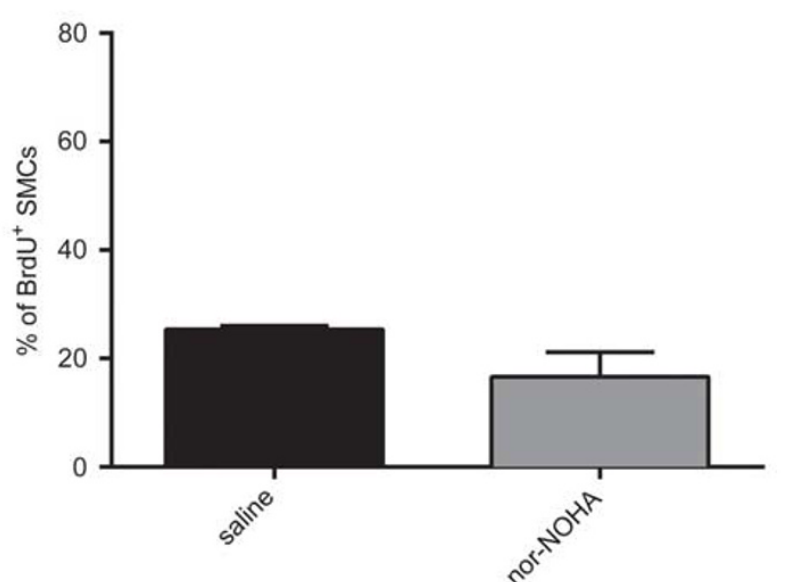




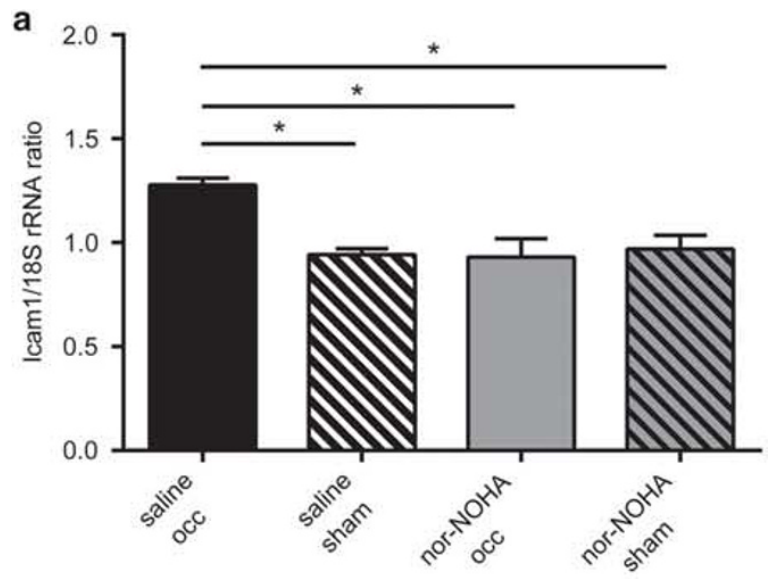

b

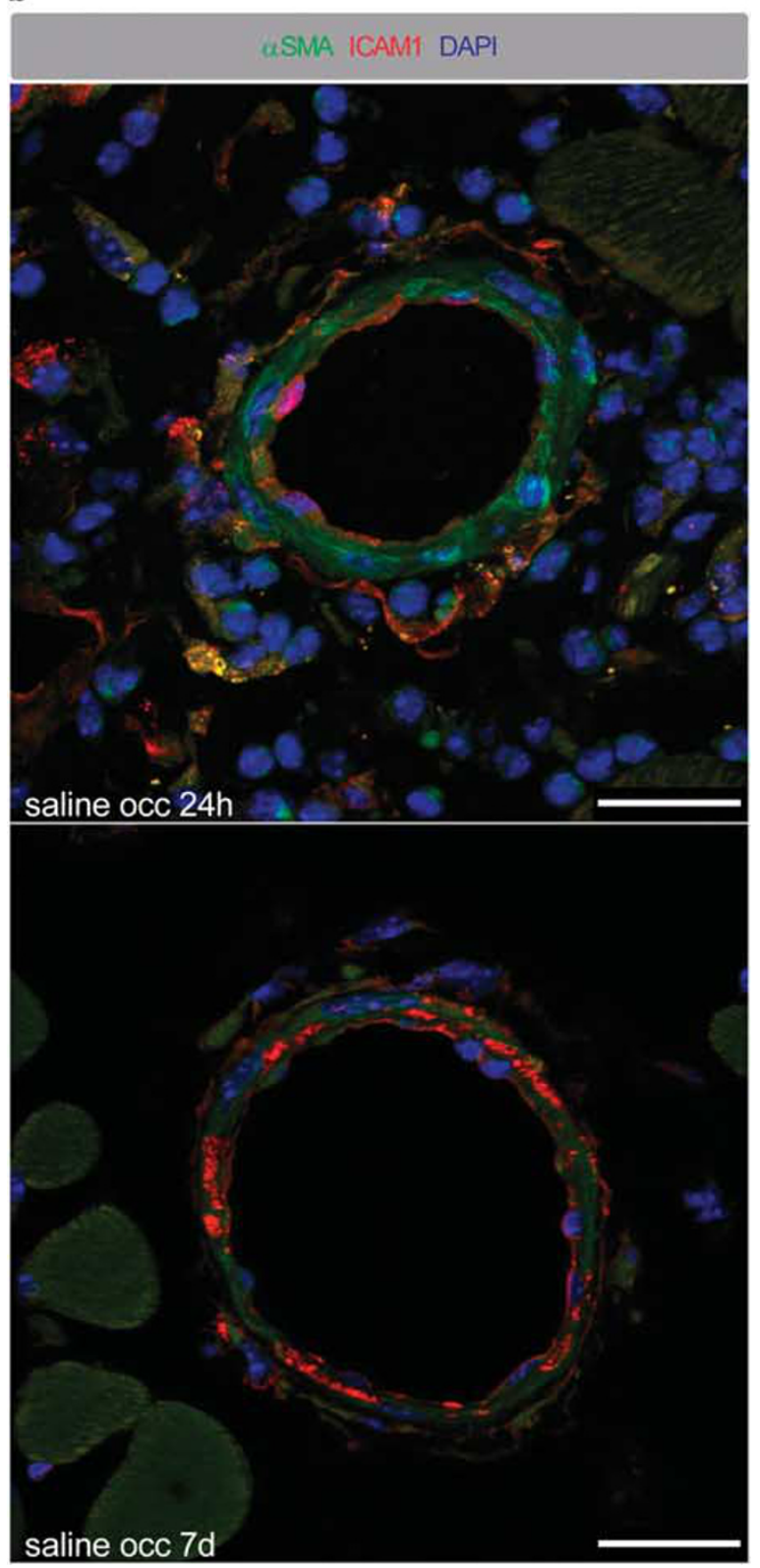

the surgical procedure until the end of the observation period. Our LDI results evidenced that treatment of mice with L-arginine did not significantly promote arteriogenesis indicating that $\mathrm{L}$-arginine is a minor rate limiting substrate for arteriogenesis under conditions of consumption of a balanced diet.

nor-NOHA is an inhibitor of arginase, but no substrate for NOS making it an ideal candidate to investigate the functional role of arginase for all kinds of physiological and pathophysiological processes. Our data showed that blocking arginase with nor-NOHA significantly interfered with EC proliferation. However, it has been shown for porcine ECs that blocking arginase under conditions of high laminar shear stress does not interfere with cell proliferation in vitro. ${ }^{25}$ Blocking arginase in primary murine arterial SMCs significantly reduced vascular cell proliferation confirming several previous observations showing particularly that arginase 1 activity is essential for SMC proliferation (for an overview see Durante ${ }^{11}$ ). For example it has been shown for rat SMCs that knockdown of arginase 1 protein arrests cells in $\mathrm{G}(0) / \mathrm{G}(1)$ phase of cell cycle and induced the expression of cyclin-dependent kinase inhibitor p21. ${ }^{26}$ However, our data evidenced moreover that blocking arginase activity resulted in increased nitrate/nitrite concentrations, indicative for increased NO production, not only in the supernatant of ECs but also of SMCs. As it is well described that NO counteracts SMC proliferation, ${ }^{10}$ we speculate that increased NO levels might have contributed to the observed reduction in proliferation of L-arginine stimulated SMCs concomittantly treated with nor-NOHA.

To investigate whether arginase activity is essential for collateral artery growth in our in vivo model, mice were treated with nor-NOHA. Laser Doppler analyses demonstrated a pronounced reduction in perfusion recovery after femoral artery ligation in mice treated with nor-NOHA compared with saline-treated mice, which served as control. Immuno-histochemical analysis evidenced a significantly reduced number of BrdU (proliferation marker) positive ECs, in nor-NOHA-treated mice, whereas the number of BrdU-positive cells was only slightly decreased. Together with our in vitro findings, these data indicate that arginase activity is essential for the process of arteriogenesis, and particularly for the proliferation of ECs in growing collateral arteries.

Vascular remodeling during arteriogenesis is well described to be promoted by perivascular macrophages. ${ }^{1,7}$ Whereas

Figure $4 \mathrm{lcam} 1$ expression in growing collateral arteries. (a) Bar chart showing qRT-PCR results on relative Icam 1 expression in collateral arteries isolated from saline or nor-NOHA-treated mice, respectively, $12 \mathrm{~h}$ after femoral artery ligation (occ) or sham operation. Data are means \pm s.e.m.; $n=3$ per group, experiments were repeated three times. ${ }^{*} P \leq 0.05$ oneway ANOVA with Bonferroni's multiple comparison test.

(b) Representative images of collateral arteries $24 \mathrm{~h}$ and on day 7 after femoral artery ligation showing ICAM1 expression. Scale bars, $25 \mu \mathrm{m}$. 
a

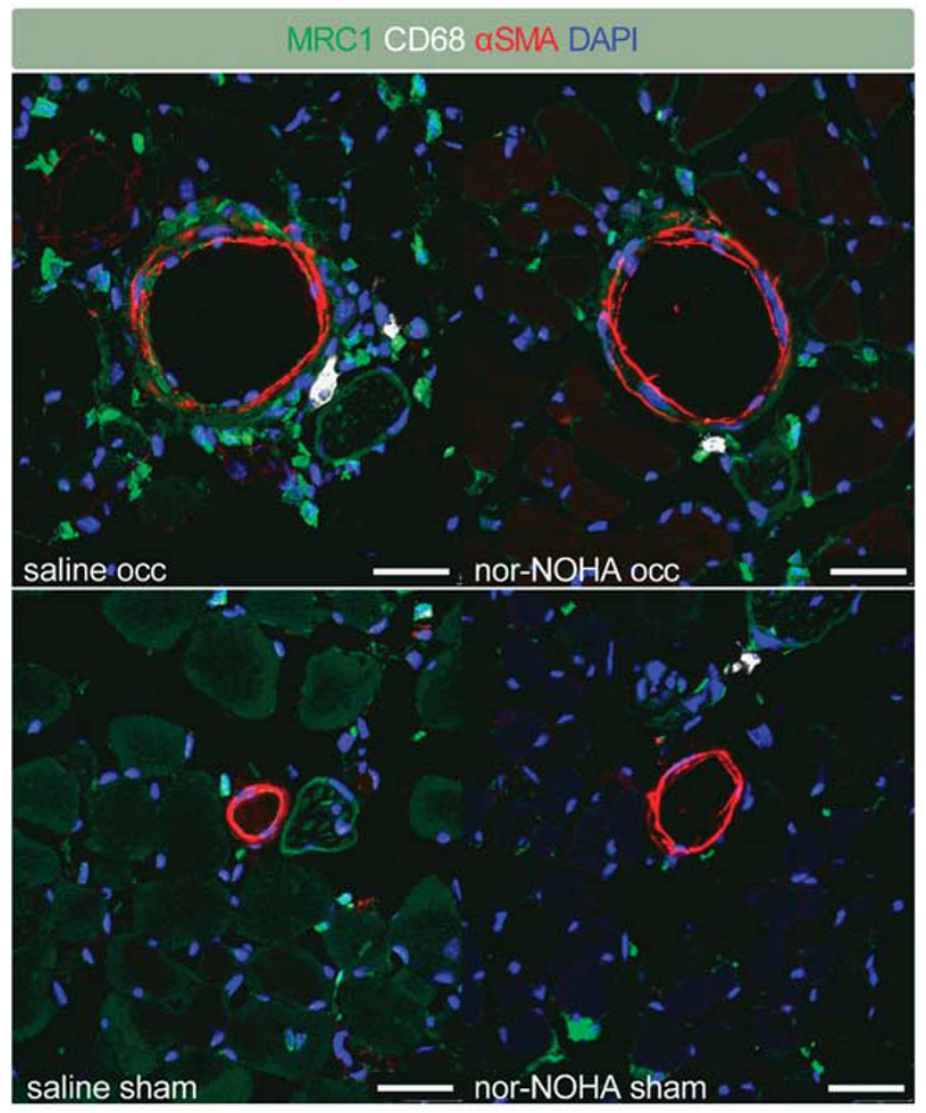

b
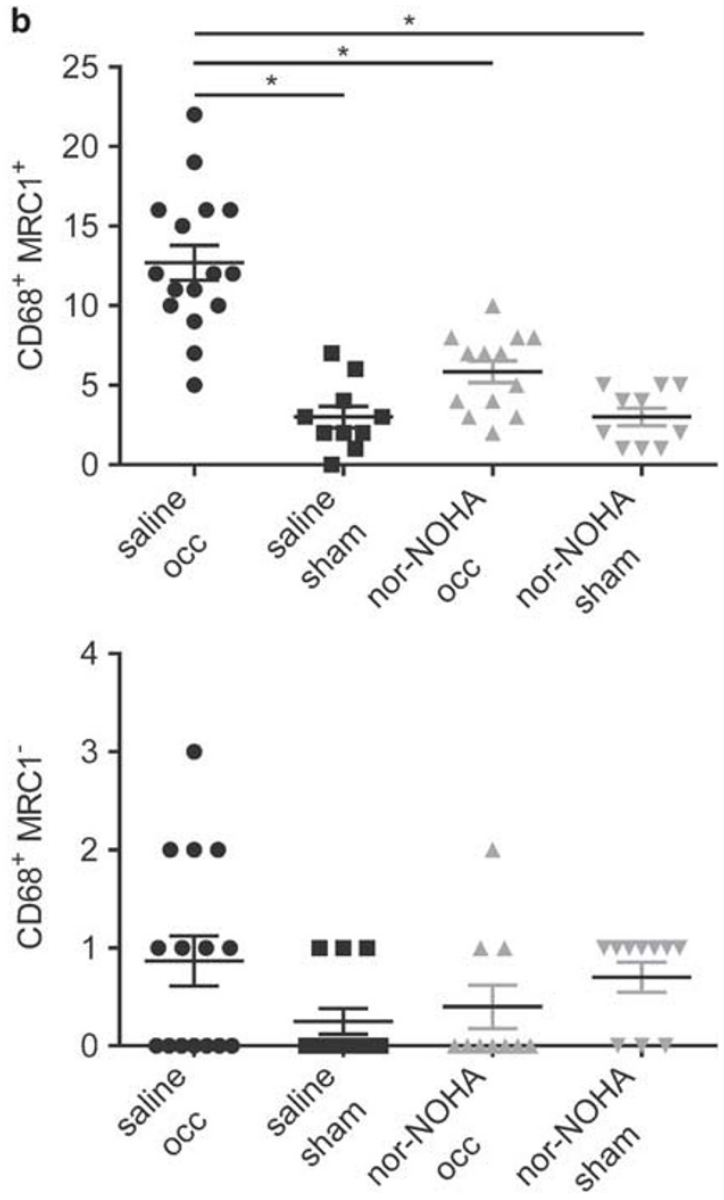

Figure 5 Quadruple staining of macrophages around collateral vessels. (a) Representative images of collateral vessels on day 7 after femoral artery ligation (occ) or sham operation and saline or nor-NOHA treatment, respectively. Scale bars, $25 \mu \mathrm{m}$. (b) Quantification of $\mathrm{CD} 68^{+} / \mathrm{MRC1}^{+}$and $\mathrm{CD} 68^{+}$ $/ \mathrm{MRC1}^{-}$macrophages accumulated in the perivascular space of collaterals. Data are means \pm s.e.m., $n \geq 10$ per group. ${ }^{*} P \leq 0.05$ one-way ANOVA with Bonferroni's multiple comparison test.

Table 1 Counts of white blood cell subpopulations of salineand nor-NOHA-treated mice

\begin{tabular}{lcc}
\hline & Saline & nor-NOHA \\
\hline Neutrophils & $0.133 \pm 0.042$ & $0.225 \pm 0.090$ \\
Lymphocytes & $0.834 \pm 0.046$ & $0.744 \pm 0.091$ \\
Monocytes & $0.013 \pm 0.002$ & $0.009 \pm 0.002$ \\
Eosinophils & $0.019 \pm 0.010$ & $0.020 \pm 0.002$ \\
Basophils & $0.001 \pm 0.001$ & $0.002 \pm 0.002$
\end{tabular}

Abbreviation: nor-NOHA, $\mathrm{N}^{\omega}$-hydroxy-nor-arginine.

Blood samples were gained 7 days after the surgical procedure. Data are means \pm s.d., $n=3$ per group. Unpaired student's $t$-test analyses gave no significances.

classically activated M1 macrophages foster inflammation and the release of proteases, M2 macrophages promote the resolution of inflammation. ${ }^{7,11}$ Interestingly, arginase 1 of alternatively activated macrophages has recently been identified to deplete arginine, thereby inhibiting inflammation. ${ }^{27}$ Hence, arginase 1 in M2-polarized macrophages might be critically involved in suppressing inflammation during arteriogenesis. In that context it is interesting to mention that arginase influence the polarization of macrophages. While arginase 2 is expressed in M1 macrophages, arginase 1 expression is a feature of alternatively activated M2 macrophages..$^{28,29}$

To investigate in more detail the functional role of arginase in collateral artery growth and its influence on macrophage recruitment and subpopulations, we performed immunohistochemical analyses and investigated differential gene expression by means of qRT-PCR. Quadruple tissue staining demonstrated a strong reduction in perivascular accumulation of M2 macrophages around growing vessels. As blood monocyte counts were not significantly changed, we assume that the reduced number of perivascular macrophages is not due to hematopoietic effects. Most of the perivascular macrophages identified in saline as well as nor-NOHA-treated animals were M2 macrophages indicating that blocking 
arginase activity does not interfere with M1 to M2 transition. However, the relative number of M2 macrophages was significantly reduced in nor-NOHA treated mice. Ming et al. have shown in human monocytes that arginase 2 silencing decreases monocyte adhesion on ECs; furthermore, that arginase 2 deficiency limits macrophage content in atherosclerotic plaques. ${ }^{11,30}$ Although it is not clear why silencing arginase in monocytes results in their reduced adhesion to ECs, one explanation might be that more L-arginine is bioavailable for NOS2, representing the major form of NOS in monocytes, and that resulting increased activity of NOS2 and hence NO production interferes with leukocyte adhesion.

A prerequisite for monocyte extravasation is their interaction with endothelial ICAM1. In recent studies, we have shown that Icam 1 is differentially expressed during arteriogenesis and that blunted Icam 1 expression as well as Icam1 deficiency is associated with less effective arteriogenesis and perivascular accumulation of macrophages. ${ }^{4-6}$ In the current study, our qRT-PCR results demonstrated that administration of nor-NOHA abolished the differential expression of Icam 1 in growing collaterals. These data are in accordance with results from Giri et al. ${ }^{31}$ showing that downregulation of arginase 2 in human umbilical vein endothelial cells abrogated leukocyte adhesion by blocking the expression of Icam 1 . Together these data indicate that arginase 2 activity in ECs is critical for perivascular macrophage accumulation, and hence effective arteriogenesis.

From our study, we conclude that arginase activity is essential for effective monocyte recruitment during arteriogenesis, and contributes to proliferation of arteriolar cells.

\section{ACKNOWLEDGMENTS}

We thank Christina Winterbauer and Christine Csapo for their excellent technical assistance. This work was supported by the Fritz Bender Stiftung (ED).

\section{DISCLOSURE/CONFLICT OF INTEREST}

The authors declare no conflict of interest.

1. Deindl E, Schaper W. The art of arteriogenesis. Cell Biochem Biophys 2005;43:1-15.

2. Faber JE, Chilian WM, Deindl $\mathrm{E}$, et al. A brief etymology of the collateral circulation. Arterioscler Thromb Vasc Biol 2014;34:1854-1859.

3. Schaper W, Pipp F, Scholz D, et al. Physical forces and their translation into molecular mechanisms. In: Schaper W, Schaper J (eds). Arteriogenesis. Kluwer Academic Publishers: Boston, Dordrecht, London, 2004.

4. Hoefer IE, van Royen N, Rectenwald JE, et al. Arteriogenesis proceeds via ICAM-1/Mac-1-mediated mechanisms. Circ Res 2004:94:1179-1185.

5. Pagel Jl, Ziegelhoeffer $\mathrm{T}$, Heil $\mathrm{M}$, et al. Role of early growth response 1 in arteriogenesis: impact on vascular cell proliferation and leukocyte recruitment in vivo. Thromb Haemost 2012;107:562-574.

6. Scholz D, Ito W, Fleming I, et al. Ultrastructure and molecular histology of rabbit hindlimb collateral artery growth (arteriogenesis). Virchows Arch 2000;436:257-270.

7. Troidl $C$, Jung $G$, Troidl $K$, et al. The temporal and spatial distribution of macrophage subpopulations during arteriogenesis. Curr Vasc Pharmacol 2013;11:5-12.
8. Kumar D, Branch BG, Pattillo CB, et al. Chronic sodium nitrite therapy augments ischemia-induced angiogenesis and arteriogenesis. Proc Natl Acad Sci USA 2008;105:7540-7545.

9. Troidl K, Tribulova S, Cai WJ, et al. Effects of endogenous nitric oxide and of DETA NONOate in arteriogenesis. J Cardiovasc Pharmacol 2010;55:153-160.

10. Madigan M, Zuckerbraun B. Therapeutic potential of the nitritegenerated NO pathway in vascular dysfunction. Front Immunol 2013;4: 174.

11. Durante W. Role of arginase in vessel wall remodeling. Front Immunol 2013;4:111.

12. Pagel Jl, Borgolte J, Hoefer I, et al. Involvement of neuronal NO synthase in collateral artery growth. Indian J Biochem Biophys 2011;48: 270-274.

13. Mees B, Wagner S, Ninci E, et al. Endothelial nitric oxide synthase activity is essential for vasodilation during blood flow recovery but not for arteriogenesis. Arterioscler Thromb Vasc Biol 2007;27:1926-1933.

14. Dai X, Faber JE. Endothelial nitric oxide synthase deficiency causes collateral vessel rarefaction and impairs activation of a cell cycle gene network during arteriogenesis. Circ Res 2010;106:1870-1881.

15. Eitenmuller I, Volger $\mathrm{O}$, Kluge $\mathrm{A}$, et al. The range of adaptation by collateral vessels after femoral artery occlusion. Circ Res 2006;99: 656-662.

16. Reisser D, Onier-Cherix N, Jeannin JF. Arginase activity is inhibited by L-NAME, both in vitro and in vivo. J Enzyme Inhib Med Chem 2002;17: 267-270.

17. Cremades A, Ruzafa C, Monserrat F, et al. Influence of dietary arginine on the anabolic effects of androgens. J Endocrinol 2004;183:343-351.

18. Eleftheriadis T, Voyatzi S, Sparopoulou T, et al. L-arginine supplementation does not affect chemically induced carcinogenesis and tumor growth in BALB-c mice. Hippokratia 2007;11:142-144.

19. Giordano E, Hillary RA, Vary TC, et al. Overexpression of ornithine decarboxylase decreases ventricular systolic function during induction of cardiac hypertrophy. Amino Acids 2012;42:507-518.

20. Limbourg A, Korff T, Napp LC, et al. Evaluation of postnatal arteriogenesis and angiogenesis in a mouse model of hind-limb ischemia. Nat Protoc 2009;4:1737-1746.

21. Burgin-Maunder CS, Nataatmadja M, Vella RK, et al. Investigation of long chain omega-3 PUFAs on arterial blood pressure, vascular reactivity and survival in angiotensin II-infused Apolipoprotein E-knockout mice. Clin Exp Pharmacol Physiol 2016;43:174-181.

22. Yang Z, Ming XF. Arginase: the emerging therapeutic target for vascular oxidative stress and inflammation. Front Immunol 2013;4:149.

23. Boulanger $\mathrm{CM}$, Heymes $\mathrm{C}$, Benessiano J, et al. Neuronal nitric oxide synthase is expressed in rat vascular smooth muscle cells: activation by angiotensin II in hypertension. Circ Res 1998;83:1271-1278.

24. Jost MM, Ninci E, Meder B, et al. Divergent effects of GM-CSF and TGFbeta1 on bone marrow-derived macrophage arginase-1 activity, MCP-1 expression, and matrix metalloproteinase-12: a potential role during arteriogenesis. FASEB J 2003;17:2281-2283.

25. Thacher TN, Gambillara V, Riche F, et al. Regulation of arginase pathway in response to wall shear stress. Atherosclerosis 2010;210:63-70.

26. Peyton KJ, Ensenat D, Azam MA, et al. Arginase promotes neointima formation in rat injured carotid arteries. Arterioscler Thromb Vasc Biol 2009;29:488-494.

27. Pesce JT, Ramalingam TR, Mentink-Kane MM, et al. Arginase-1expressing macrophages suppress Th2 cytokine-driven inflammation and fibrosis. PLoS Pathog 2009;5:e1000371.

28. Khallou-Laschet J, Varthaman A, Fornasa G, et al. Macrophage plasticity in experimental atherosclerosis. PLoS ONE 2010;5:e8852.

29. Martinez FO, Helming L, Gordon S. Alternative activation of macrophages: an immunologic functional perspective. Annu Rev Immunol 2009;27:451-483.

30. Ming XF, Rajapakse AG, Yepuri G, et al. Arginase II promotes macrophage inflammatory responses through mitochondrial reactive oxygen species, contributing to insulin resistance and atherogenesis. J Am Heart Assoc 2012;1:e000992.

31. Giri $\mathrm{H}$, Muthuramu I, Dhar M, et al. Protein tyrosine phosphatase SHP2 mediates chronic insulin-induced endothelial inflammation. Arterioscler Thromb Vasc Biol 2012;32:1943-1950. 